\title{
DIRECT CHAOTIC COMMUNICATIONS AND ACTIVE RFID TAGS FOR INTERNET OF THINGS AND INTERNET OF ROBOTIC THINGS
}

\author{
Alexander S. Dmitriev, Anton I. Ryzhov, Maxim G. Popov \\ Kotelnikov Institute of Radioengineering and Electronics of RAS, http://www.cplire.ru \\ Moscow 125009, Russian Federation \\ chaos@mail.cplire.ru
}

\begin{abstract}
The use of ultra-wideband direct-chaotic communications and radio identification tags for the organization of wireless high-speed data transmissons on the Internet of things and the Internet of robotics is considered. Prototypes and models of ultra-wideband transceivers of the 3.0 - 5.0 GHz range are described and presented. It is noted that these transceivers and nodes based upon them are intended for usage in sensor and active networks with arbitrary topology. It removes some of the limitations inherent for a number of other solutions for communication and identification.
\end{abstract}

Keywords: ultrawideband communications, internet of things, radiofrequency identification, direct chaotic communications

UDC 621.391

Bibliography - 19 references

Received 03.09.2018

RENSIT, 2018, 10(2):313-322

DOI: $10.17725 /$ rensit.2018.10.313

\section{CONTENT}

1. INTRODUCTION (313)

2. INTERNET OF THINGS AND "SMART SPACES" (314)

3. REQUiREMENTS FOR WIRELESS COMMUNICATIONS (315)

4. Technical communication EQuipment (317)

5. UNIVERSAL MODULE AND NETWORK NODES BASED ON IT (319)

6. Active ultrawideband tag (320)

7. Conclusion (321)

REFERENCES (322)

\section{INTRODUCTION}

The Internet of Things (IoT) is rapidly evolving from the conceptual point of view, extending the scope of applications, searching for and developing suitable wireless technologies. In the last few years an important part of the Internet of things has been realized. It is associated with remote data collection (kilometers and tens of kilometers) of simple data from electric and water meters in housing and communal services and simple management, for example, for electric lighting in cities and on the routes $[1$,
2]. On the other hand, direction which defines the concept of the Internet of things as smart houses also continues its growth. Just from these two examples it becomes clear that the requirements to the Internet of things depend on situation and they are very different both in the transmission speed and in the distances to which information is to be delivered. In particular, in smart homes these distances can be up to several dozens meters or up to one hundred meters at maximum. And there are also such areas of the Internet of things as industrial Internet, agriculture, transport, logistics centers, robotics, etc. In [3] the Internet of things specifically highlighted the concept of the Internet of robotics (Internet of Robotic Things - IoRT). It refers to such objects as "... intelligent devices capable of tracking events by combining sensor data from different sources, using local and distributed "intelligence" to determine the best actions, and then to manipulate or monitor the objects in the physical world, and in some cases, to physically move through this world for the action itself... ".

Vivid example for the system of IoRT objects is unmanned automobile transport. According 
to some estimates, as early as 2020, around 250 million connected cars will travel the roads around the world [4]. In the next five years, the number of cars equipped with communications equipment will grow dramatically, making connected cars one of the main elements for the Internet of things. Every fifth car in the world will have a wireless network connection with other devices. At the same time wireless communications inside the cars themselves quickly spread from luxury models to middleclass ones.

The article discusses the concepts of IoT and IoRT, the possibilities that can be implemented for the Internet of things with the use of wireless ultrawideband direct-chaotic communication facilities at the physical level [5] and Radio Frequency Identification (RFID) in the range 2.85-10.6 GHz. It also presents experimental prototypes and models of ultrawideband directchaotic transceivers and active RFID tags of the $3.0-5.0 \mathrm{GHz}$ range with such characteristics. It should be noted that these transceivers and tags are intended for use in sensor and active networks [6] with arbitrary topology, which removes a number of limitations. typical for a number of other solutions. Using adaptive algorithms, it is possible to change the speed and range of transmission in the same network in order to provide solutions for different types of tasks.

\section{INTERNET OF THINGS AND "SMART SPACES"}

A number of concepts that overlap in meaning, but have their own specific features, are associated with with the Internet of things. To avoid confusion, we begin by clarifying the terminology [7].

In the early 70 's, the inventors of the Internet drew in the imagination a world in which networks would connect to each other, becoming a web of interacting systems. By 1973, it became obvious that the main thing was to figure out how to make computers exchange information.
By that time were developed various computer networks had been developed, which worked independently of each other. However, the value of all these systems was very limited, since they could not exchange information. To solve this problem, the TCP/IP (Transmission Control Protocol/Internet Protocol) was invented with which made it became possible to connect computers and all kinds of computer networks. The basis of the connected world was laid. Today every device connected to the Internet gets its IP address, and this allows it to connect to other devices.

In the phrase "Internet of things", "things" literally mean things that connect to the Internet and to each other. Each of these connected objects has a unique identification number and IP address. The connection can be made using wires, cables or wireless communication. The Internet of things implies the possibility of moving data needed for process control to any distance - both from another room and from another part of the world.

Within such broad category as the Internet of things, there are some key differences and nuances. Therefore, it is useful to introduce basic definitions.

The term "connected devices" refers to devices that exchange data over a conventional Internet connection, or are connected to a common network, for example, closed or private network. The connected device does not necessarily connect to the Internet of things, but it happens more often. In addition, this connectivity is spreading farther and farther, going beyond computers and penetrating into all corners and corners of the world.

There are two main types of connected devices - they are divided into physical and digital [8]. The first group includes such objects and processes that do not themselves generate or transmit digital data unless special manipulations are made and no changes happened. The second group includes devices that, according to their purpose, are capable of generating data and 
transmitting them for future use. Therefore, despite the fact that many physical objects can be tagged using digital technologies, such as passive RFID tags, they are not capable of generating and transmitting any detailed meaningful data.

At the same time, RF identification is the main tool that allows physical devices to enter the digital world via wireless communication channels. RFID tags can be active and passive. Both of them allow nearby located readers to collect data and exchange them with a computer.

Another term related to the Internet of things is "Industrial Internet", concerning machines equipped with sensors that make them "smart". In the field of industrial Internet, data exchange is usually performed in three different ways: machine-machine (M-M, M2M), humanmachine (H-M, H2M), and human-smartphone (M-C, M2S) (or other devices, for example, the tablet).

The Internet of things is able to connect together primarily physical objects and items, and also connect them with digital devices, including computers and software applications.

Thus, all devices interact with each other within a group or multi-point configuration and exchange data in real time - often using cloud technologies. When all these machines are connected to people using various computing devices, a completely new conceptual basis arises - the "Internet of everything". The Internet of everything is a more developed and perfect structure, in which the physical and digital worlds merge into a single space.

Currently, most of the data presented on the Internet in the form of text files, messages, audio and photo and video files. The Internet of things collects different new data, unites them in many ways and gives people and machines a broader and deeper understanding of the processes. To do this, you need to give computers their own tools for collecting information so that they can see, hear and smell the world themselves [9]. The Internet of things is able to penetrate into all the nooks and corners, cracks, holes and wormholes existing in an inaccessible for perception, and often an invisible world that extends far beyond the abilities of human organs for sight, hearing, smell and consciousness. It creates new types of networks and systems - completely new routes for data, information and knowledge.

\section{REQUIREMENTS FOR WIRELESS COMMUNICATIONS}

How the Internet of things works from the communication and network point of view? And what in this sense determines its power as a technology?

Two points are emphasized in [9] concerning the network nature of the Internet of things' power:

- Connecting a separate device increases its power, and often also the value for the user. However, the ability to connect a device to an extensive network - in fact, to the Internet of things - causes technical capabilities to grow exponentially.

- The benefit of connected devices is not the ability to start the engine or adjust the temperature in the house using the smartphone application. The real benefit will come when the whole networks of devices will exchange data and apply them in practice. As a result, the products of technology evolution will make a revolution.

These IoT properties fully comply with the laws for networks that characterize their capabilities. There are three such laws: Sarnov's law, Metcalfe's law and Reed's law [10].

Sarnov's law was formulated with the emergence of radio and television networks in the early 20th century, when the broadcast to multiple receivers came from a small number of transmitting stations. One of the pioneers of broadcasting David Sarnov stated the obvious: "The value of broadcast networks is directly proportional to the number of their listeners and viewers."

The Law of Metcalfe determines the growth of the value of the network in the presence of 
connections between its nodes. The total value for the network, where each node is accessible to all other nodes, increases as the square of the number of its nodes. If you have two nodes and the value of each one is one unit, then the value of the network merged from them becomes four. Four interconnected nodes with value of one unit, within the network gain a total value of sixteen units. And the value of hundreds of such nodes is a hundred times a hundred, that is, ten thousand. This mathematical consequences can be expressed economically: the connection of the two networks gives a value significantly greater than the value obtained by adding their values as independent networks.

Reed's law shows that the value of a network increases not as a square, but as an exponent, if it is possible to form arbitrary groups within the network for exchanging information with each other.

What is significant is that the value of a network moves from one category to another as the concept of network expands. Whether growth is due to the gradual addition of consumers or the transparency of the relationships, the degree of expansion is such that it can support new categories of "market invaders", and hence new competitive games.

A similar, expansion-driven value shift can be observed in the history of the Internet. Initially, the use of the Internet was dictated by its role as a network of terminals, which shared selective access to a small number of expensive main hosts. As the Internet grew, its value and use increasingly concentrated around paired exchange of electronic messages, files and so on, increasing its value in accordance with the law of Metcalfe. And with the beginning of the 1990s, the flow of data between teleconferences and Web sites, mailings, and so on began to dominate the Internet, increasing value in accordance with the exponential law for networks with the ability to create groups. Although the functions that prevailed before did not lose their value and did not diminish as the Internet grew, the value and use of services, determined by the prevailing laws of correspondence, grew significantly faster. Therefore, many types of contacts and cooperation outside the Internet have been absorbed by the expanding functions of the Internet, which has become a new sphere of competition.

It is natural that in the early stages of the development of the Internet of things, "objects" will be connected to certain centers-nodes of the network and their direct interaction will be set to a minimum. These objects, except for "things" with active and passive RFID tags, will be sensors, that can collect and transfer some data to the nodes, and actuators, that can transmit control commands to "things". The task of informational interaction between the "things" will arise only when "thing" will need information from other devices to analyze the situation and make some decisions. So such a "thing" should have at least some minimal intellectual resource in the form of, for example, a processor, through which it can carry the analysis.

Although various communication channels, including cable and wires, can be used on the Internet of things, we will be considering radio channels and networks based on wireless transceivers as the most in-demand. Then we can assume that in the first stage of Io'T development the most widespread will be wireless local area networks with the topology of the star type, where the transceivers of "things" in the local network interact with a certain node, and the direct interaction between the transceivers inside the local network will be minimal. In this case, the contribution of local networks of Internet of things will grow in proportion to the number of objects connected in these networks. And in the general dependence for potential "strength" of the network, an additional linear factor will appear.

With the growth of the number of "things" in the local network, there inevitably will be a situation in which "things" will begin to interact. For example, it can be an intelligent sensor 
network for monitoring certain parameters of a territory, say, a temperature and it can independently estimate the temperature situation in the territory by exchanging data between devices and pass the results of this analysis to the decision node. In this case, the topology of the local wireless network can vary, depending on the location of the sensors and the range of the radio facilities. In general, it would be desirable for the network to allow the implementation of any topology and at the same time to have the capabilities of self-organization. In this case, the contribution of local network for Internet of things will grow in proportion to the square of the number of objects connected in this network. In the general dependence of for potential "strength" of the network, an additional quadratic multiplier will appear.

Finally, if the "things", like the objects of the classical Internet, can participate in the formation of arbitrary groups exchanging information with each other, Reed's law will begin to work and the strength of the network will grow exponentially with the total number of objects on the network.

Is there something specific for the Internet of things and the Internet of robotics depending on the number of objects in them? Formally, as we can see, the same laws as in the classical Internet work here. However, there must be some physical limitations on exponential growth. Indeed, there are at least two such restrictions.

The first thing is related to the cost of nodes in the network. Increase in the number of nodes in the Internet of everything (in the combined classical Internet with the Internet of things) will mainly occur due to the Internet of things. And the growth in the number of objects in it will be determined by economic and technological restrictions stemming from Moore's law. Suppose that the number of objects in the Internet of things is more than 10 times the number of objects in the classical Internet. This can happen in a limited time only if the cost of each IoT object is significantly lower than the cost of equipment in a typical node. Lets say it should decrease by 5-10 times. If this number increases tenfold again, the unit cost should decrease by 5-10 times more. If we assume that the cost of the classical Internet node equipment is $\$ 500$, then an increase in the number of nodes will be 10 times when the node cost is reduced to 50 $100 \$$, and 100 times when the cost decreases to $1-4 \$$.

The second limitation is related to the physical volume in which nodes for the Internet of things can be placed. Let's take the volume near and inside the Earth, in which all kinds of sensors and objects of the Internet of things can be placed. This volume is equal to the surface area of the Earth, multiplied by the thickness of the layer in which objects can be located. Let, for definiteness, it is equal to $100 \mathrm{~m}$. Then the total volume filled with objects of the Internet will be $h=5 \cdot 10^{7} \mathrm{~km}^{3}=5 \cdot 10^{16} \mathrm{~m}^{3}$.

If we assume that the volume of the usual Internet is about 10 billion, then with a volume of the Internet of everything 100 times more than the classical Internet, the number of objects will be $10^{12}$. Or one object at $5 \cdot 10^{4} \mathrm{~m}^{3}$ - in a cube with a side of about $40 \mathrm{~m}$. Given the strong uneven distribution of objects, their extreme density can reach 1 object per $1 \mathrm{cu}$. $\mathrm{m}$ and above.

\section{TECHNICAL COMMUNICATION EQUIPMENT}

The technical communication equipment of the Internet of things, the Internet of robotics and the Internet of everything include transceivers, sensors, actuators, tags, readers, storage databases (including cloud databases) and software with a hardware on which it is located. And the capabilities of both individual identification points (nodes) and identification systems as a whole will be determined by the capabilities and characteristics of RFID tags and readers.

Let us briefly consider the main types of radio tags and their characteristics.

We will be primarily interested in wireless communication and radio identification devices operating in the microwave frequency range. 
This is due to the potential need to transfer large amounts of data combined with the requirement for small size of the devices themselves (tags) that are required for most mass applications. Ultra-wideband tags should operate in the range from 2.85 to $10.6 \mathrm{GHz}$ and therefore it is important to compare their characteristics with narrowband devices in approximately the same frequency range.

In general there are three types of tags can be used in the Internet of things: passive, semipassive (semi-active) and active. However, they perform and will perform different functions in the IoT and IoRT due to their technical characteristics. Table $\mathbf{1}$ compares the basic properties of active and passive technologies and their capabilities. It can be seen that the main limitations for passive tags are a small range and small amounts of transmitted information. The latter makes it difficult to use passive radio identification to connect devices of the second type - digital devices that generate information or process information while collecting data from sensors. On the other hand, the significantly greater range and data volume capabilities of active RF tags are not free. They are provided by much greater cost of devices and the presence

Table 1 Comparison of active and passive RFID technologies

\begin{tabular}{|l|l|l|}
\hline \multicolumn{1}{|c|}{ CRITERION } & \multicolumn{1}{|c|}{ ACTIVE } & \multicolumn{1}{c|}{ PASSIVE } \\
\hline Power source of a tag & Internal, on a device & $\begin{array}{l}\text { Energy is transmitted } \\
\text { from reader through } \\
\text { radio channel }\end{array}$ \\
\hline Power source on tag & Yes & No \\
\hline $\begin{array}{l}\text { Power availability on } \\
\text { tag }\end{array}$ & Constant & Only during reading \\
\hline $\begin{array}{l}\text { Power availability on } \\
\text { tag }\end{array}$ & Low & High \\
\hline $\begin{array}{l}\text { Strenght of the tag signal } \\
\text { on the reader }\end{array}$ & High & Low \\
\hline Communication range & $\begin{array}{l}\text { Long (up to 100 } \mathrm{m} \\
\text { and more) }\end{array}$ & $\begin{array}{l}\text { Short or very short (3 } \mathrm{m} \\
\text { or less) }\end{array}$ \\
\hline Number of tags in area & $\begin{array}{l}\text { More than 1000 tags } \\
\text { for 1 reader, possible } \\
\text { to serve 20 tags while } \\
\text { moving with a speed } \\
\text { more than 100 kmph }\end{array}$ & $\begin{array}{l}\text { Hundreds of tags within } \\
\text { 3 meters around reader, } \\
\text { can serve 20 tags while } \\
\text { moving with a speed 3 } \\
\text { kmph or lower }\end{array}$ \\
\hline Sensor possibilities & Yes & Limited \\
\hline $\begin{array}{l}\text { Memory capacity } \\
\text { available for writing/ } \\
\text { reading }\end{array}$ & $\begin{array}{l}\text { High (for example } \\
128 \text { Kb) }\end{array}$ & $\begin{array}{l}\text { Low (for example, 128 } \\
\text { bytes) }\end{array}$ \\
\hline
\end{tabular}

of power source with limited battery life on the tags.

These circumstances determine now and will determine in the future the scope of application for active and passive radio identification in the basis of the Internet of things. Note that current popularity of active tags is connected mainly with their ability to determine the location of tags with sufficiently high accuracy. Ultrawideband tags and technologies provide the better results for solving such problems and they successfully developing.

With the development of IoT and IoRT technologies, the situation will change in favor of systems that will be required to collect and deliver large amounts of data (at least under peak loads). Such systems will require tags as universal devices that can identify the objects on which they are located, enable to determine their position in space, collect information (for example, from sensors), process it and transmit it to the reader or neighboring nodes. So the tag will be transformed from the narrow purpose device into a universal information and communication node. At the same time, the cost of such devices should gradually decrease, and the battery life should be sufficient to solve the corresponding problems.

Further, as a candidate for the role of such devices, direct chaotic ultrawideband universal transceiver modules capable, among other things, to act as active labels, are considered.

Direct chaotic transceivers [11-13] were created oriented to the standard IEEE 802.15.4a - 2007 [14], which regulates the use of ultra-wideband signals in personal wireless networks at the physical level (Ultra Wideband Personal Area Networks, Physical level). In this standard, direct chaotic signals (chaotic radio pulses) are used as an optional data carrier. In the following years, there was a high level of activity in the UWB communication standards development by the IEEE (IEEE 802.15.4 2011, IEEE 802.15.6 - 2012, IEEE 802.15.4f - 2012), the International Organization for 
Standardization (ISO) and the International Electrotechnical Commission (IEC) (ISO / IEC 24730-61 - 2013, ISO / IEC 24730-62 - 2013).

The IEEE 802.15.4 - 2011 standard refers to the physical layer of general purpose personal networks, integrating and upgrading the IEEE 802.15.4 - 2006 and IEEE 802.15.4 - 2007 standards. The IEEE 802.15.6 - 2012 standard refers to medical and consumer networks located near the body of a person (body networks). Standards IEEE 802.15.4f - 2012, ISO / IEC 24730-61 - 2013, ISO / IEC 24730-62 - 2013 define the requirements for ultrawideband active radiotags. It is interesting to note that the data transfer rate in the IEEE 802.15.4a-2007 standard is limited to $200 \mathrm{Kbps}$, but the IEEE 802.15.4-2011 standard created on its basis introduces the transmission rates of $110 \mathrm{Kbps}$, 850 Kbps, 6.8 Mbps and 27.2 Mbps. The same transmission speeds appear in the ISO / IEC standard 24730-62 - 2013.

After the adoption of the standard for active tags (IEEE 802.15.4f), the first devices appeared on the market provided, among other things, the possibility of high-precision location of labels. These devices include Zebra UWB radio tags and readers [15] and tags from Trackit Systems [16]. Another interesting UWB product is DecaWave's UWB transceiver chip and a transceiver module based on it [17]. The company also supplies a set for development based on the chip.

Note that these practical foreign developments of UWB communications mainly focus on the task of range determination and object localization, while the actual task of data transmission is given less attention. Ultrawideband communication devices based on chaotic radio pulses, in contrast, are mainly designed to solve problems of information transfer and transmission of data streams over networks. Therefore, the requirements for these two areas of UWB wireless communication equipment are significantly different.

\section{UNIVERSAL MODULE AND NETWORK NODES BASED ON IT}

Considering the development trends of ultrawideband communication, the characteristics of the developed transceivers based on chaotic radio pulses and the experience gained at the Kotelnikov Institute of Radioengineering and Electronics (IRE) of RAS, a universal UWB direct chaotic transceiver module was developed [6], designed to solve a wide range of problems in the field of short range wireless communication.

The developed ultrawideband direct chaotic transceiver module PPM-47 is a smallsized device that can be connected to sensors, actuators, batteries and other components on special boards.

The module structure is shown in Fig. 1. It includes an ultrawideband transmitter (UT) unit, an ultrawideband receiver (UR) unit, an antenna, a key $(\mathrm{K})$, and a digital unit (CPU). CPU provides control of data transmission and reception, implements network functions and interface for connection to external devices. Such devices can be sensors or actuators made on separate boards. External devices can be either digital or analog. For the analog ones a built-in analog-todigital converter of the microcontroller can be used to convert the signal to digital form. The microcontroller used in a transceiver is a highperformance 32-bit STM232L with reduced power consumption. Digital transmission between the microcontroller of the transceiver and external device is carried out via a highspeed SPI interface. The module can provide information transfer between two devices in the point-to-point mode, as well as by the network. In the case of network transmission all control tasks for the network level are provided by the microcontroller of the device itself.

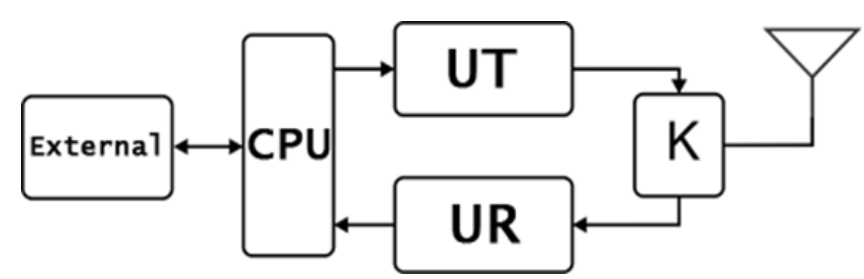

Fig. 1. Structure of UWB transciever module. 
Structurally, the module is a board of $50 \times 35 \times 6 \mathrm{~mm} 3$ size, made on a FR-4 material with a thickness equal to $0.6 \mathrm{~mm}$.

The operating frequency range of the transceiver module is within $3-5 \mathrm{GHz}$, the physical transmission rate is up to 6 (12) Mbps.

The module can be used independently, without special additional boards or devices, for example, as a base station, connected to a computer or as a relay. It can also be part of sensor and actuator nodes, where sensors or actuators are present with it.

In Fig. $2 a$ shows the base station based on the module. It has a matchbox form-factor and connects via a micro-USB connector to a computer, laptop or smartphone. The device is powered by a micro USB connector. The use of various additional blocks in the form of specialized boards enables the realization of a wide range of devices. For example, such blocks can be: a board with a rechargeable battery, which makes it possible to use the module as a relay (Fig. 2b), a board with a disposable battery (Fig. 2c), which allows o use the module as the active ultrawideband tag and a board with an acoustic module (Fig. 2d).

Relay node - can be used when it is necessary to increase the communication range. Along with the universal module includes a board with the battery. Battery can be charged is via the microUSB connector (Figure 2b).

Active RFID tag - a device that provides identification for the object to which it is attached. An active RFID tag, in addition to the universal transceiver module, includes a power supply board with a $600 \mathrm{mAh}$ lithium battery,

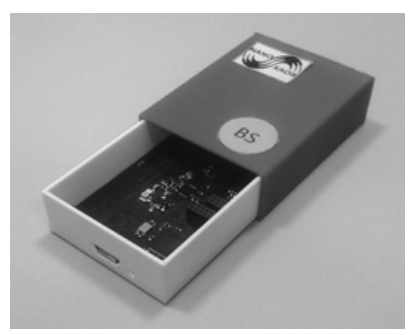

(a)

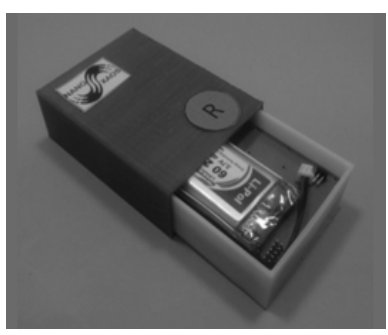

(b)

which allows the tag to operate autonomously for two years or more (depending on the operating mode). The tag, like the other devices based on the universal module, is placed in a plastic case with the matchbox form-factor(Fig. 2c).

Acoustic sensor node - can be used for personal voice communication between people in the office or at home. It can be called a "remote voice". Along with the universal module, it includes a board with a microphone, a speaker, a microcontroller and a battery. The battery is charged via the micro-USB connector. The acoustic sensing unit, like the tag, is placed in a plastic case with the form-factor of a matchbox (Fig. 2d).

\section{ACTIVE ULTRAWIDEBAND TAG}

As an example of a node of a direct chaotic network, let us consider in more detail the active ultrawideband tag.

Different versions of tags are the basis for the concept of the Internet of things and the Internet of everything $[18,19]$.

The tag based on chaotic radio pulses can work in two modes:

Beacon mode (Fig. 3a). In this case, the label periodically sends the information it contains in the form of packets. The frequency of sending can vary from 0.01 packets per second to 10 packets per second.

Response mode (Fig. 3b). Information is sent as a packet in response to a request from an external device, such as a reader.

The tag works in conjunction with the root node (reader). The reader uses the same transceiver module as the tag. This module can work with the internal antenna or with the external

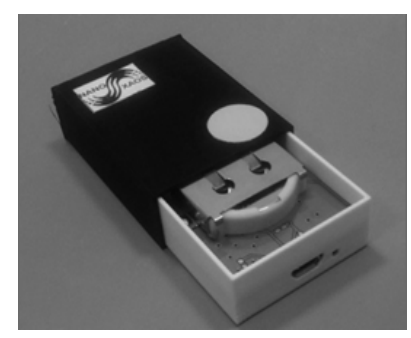

(c)

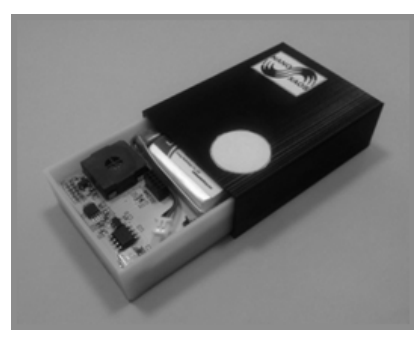

(d)

Fig. 2. Nodes of active UWB network based on universal module: base station (a); relay node (b); active RFID tag (c); acoustic sensor node (d). 


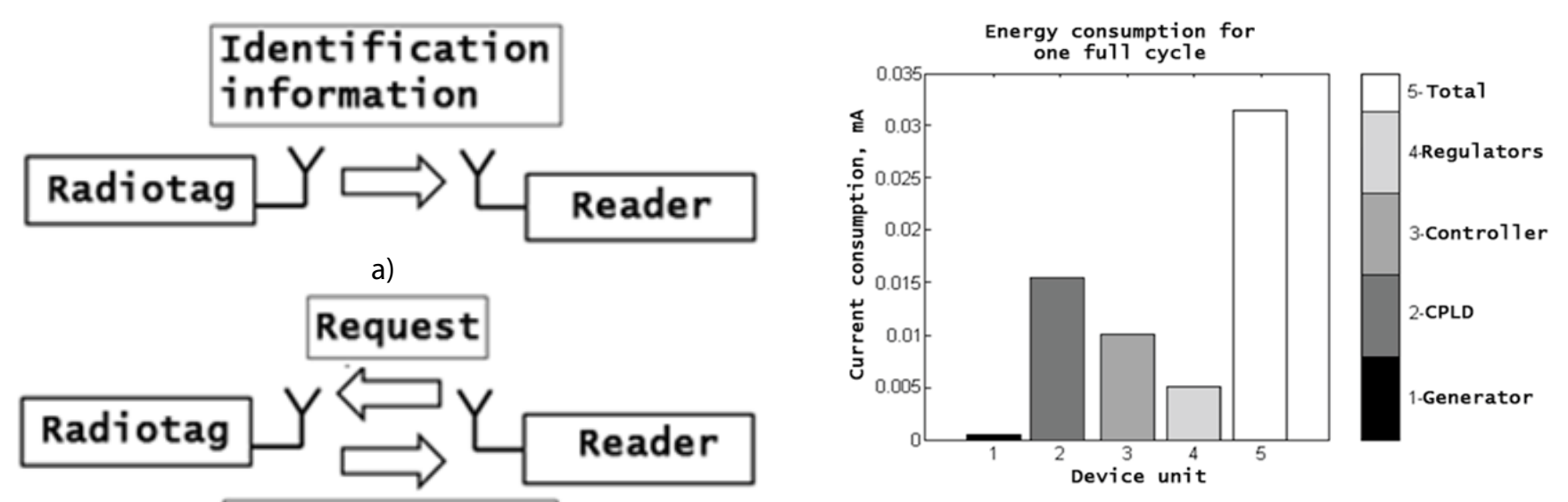

Identification information

b)

Fig. 3. Interaction scheme for tags and readers: beacon mode (a); response mode (b).

one. In the first case, the communication range is up to 30 meters. And if an external directional antenna is used in the reader, the range can be increased up to 100 and more meters.

To ensure long-term battery life, the device implements the system of energy-saving modes.

Short packet length combined with a high transmission rate allows simultaneous operation in the beacon mode for a large number of tags without any coordination. For example, 1000 radio tags with a packet transmission length of 17 microseconds (10 bytes packet size) and packet sending interval of one second will give the probability of collision between packets $\sim 10^{-2}$ in the region of stable reception.

As a power source for the tag a lithium battery CR2450 is used. The battery has an output voltage of $3 \mathrm{~V}$ and is designed for small values of the output current, $2 \mathrm{~mA}$, at which excess capacity of the battery begins to significantly drop. The transceiver is powered by a voltage of $5 \mathrm{~V}$, and the peak current consumption can be up to $45 \mathrm{~mA}$. Considering these factors, a special electrical circuit is used to power the radio tag. It utilizes capacitors to accumulate the charge in pauses between data transmissions and provides the required current at the time of transmission or reception.
Fig. 4. Energy consumption of the device: 1 - generator; 2 CPLD; 3 - microcontroller; power regulators; 4 - the whole tag.

When the radio tag operates in the beacon mode, it sends a message containing the identification information with some constant period. Then the message can be is received and processed on the reader. The overall duty cycle of the radio tag look like this. For the most of the time of its operating cycle the device is in sleep mode, but it periodically wakes up for the time required to send the packet. Transitions between modes are controlled by the microcontroller. In the sleep mode, the entire periphery of the device, with the exception of the power regulators, is turned off. The microcontroller stops all operations and counts the time until the next packet. At the end, it switches to the operation mode and sequentially turns on all the necessary peripherals. After sending the identification packet, the microcontroller turns off all the peripherals and switches back to the time counting mode until the next packet.

The structure of power consumption with a breakdown by units for the beacon mode with a period of 1 second is illustrated in Fig. 4 . Calculation of the average power consumption gives a value about $30 \mu \mathrm{A}$, with peak consumption at the moment of transmission up to $45 \mathrm{~mA}$.

\section{CONCLUSION}

Analysis of development prospects for the of Internet Things and the Internet of everything shows that their strength will be determined by the number of "things" that interact at short distances. In order to enable for the value of 
the Internet of everything to increase at least proportionally to the square of the number of connected "things", wireless short-range communication networks which can implement any topology and at the same time provide a sufficiently high data transfer rate are needed. In addition, they should be capable of longterm autonomous work, even in situations with a changeable network topology (as in the case of mobile robot groups), and also to work in conditions of increased range for certain cases.

Wireless UWB direct chaotic communication devices meet the above requirements and can provide Io'T and IoRT with a wide range of capabilities, so they can be considered as perspective platforms, both for general-purpose nodes and for active RFID tags.

The study was carried out at the expense of a grant from the Russian Science Foundation (project No. 16-19-00084).

\section{REFERENCES}

1. Tikhvinsky V, Koval V, Bochechka G. Lora Technology: Prospects of Implementation on IoT Networks. First Mile, 2016, 6:43-49.

2. Zhdanov A. "LoRa," Swift "or Sigfox". TeleSputnik, 2017, 256(2):48-49 (in Russ.).

3. The Internet of Robotic Things. https:// www.abiresearch.com/marketresearch / product/1019712-the-internet-of-roboticthings/ (accessed October 12, 2016).

4. Gartner Says By 2020, a Quarter Billion Connected Vehicles Will Enable New In-Vehicle Services and Automated Driving Capabilities. http: / / www.gartner.com/ newsroom / id/2970017 (accessed October 12, 2016).

5. Dmitriev AS, Efremova EV, Gerasimov MY. Multimedia sensor networks based on ultrawideband chaotic radio pulses. J.Commun. Technol. El., 2015, 60(4):393-401.

6. Dmitriev AS Gerasimov MY, Itskov VV, Lazarev VA, Popov MG, Ryzhov AI. Active wireless ultrawideband networks based on chaotic radio pulses. J.Commun. Technol. El., 2017, 62(4):380-388.
7. Greengard S. The Internet of Things MIT Press Essential Knowledge series). Cambridge, Massachusetts, MIT Press Publ., 2015, p 232.

8. Internet of Things vs. Internet of Everything What's the Difference? ABI Research, 2014:1-10.

9. Ashton K. That 'Internet of Things' Thing. RFID Journal, 2009, https://www.rfidjournal. com/articles/view?4986.

10. Rheingold H. Smart mobs: The Next Social Revolution. Cambridge, Basic Books, 2003, p. 288.

11. Dmitriev AS, Efremova EV, Kletsov AV, Kuzmin LV, Laktyushkin AM, Yurkin VY. Wireless ultrawideband communications and sensor networks. J.Commun. Technol. El., 2008, 53(10):1206-1216.

12. Dmitriev AS, Ryzhov AI, Lazarev VA, Mansurov GK, Popov MG, Malyutin NV. Experimental ultrawideband wireless sensor network for medical applications. J.Commun. Technol. El., 2015, 60(9):1027-1036.

13. Dmitriev AS, Efremova EV, Gerasimov MY. Multimedia sensor networks based on ultrawideband chaotic radio pulses. J.Commun. Technol. El., 2015, 60(4):393-401.

14. IEEE Standards Association, «802.15.4a-2007 - IEEE Standard for Information technology-Local and metropolitan area networks-- Specific requirements-- Part 15.4: Wireless Medium Access Control (MAC) and Physical Layer (PHY) Specifications for Low-Rate Wireless Personal Area Networks (WPANs) Amendment 1: Add Alternate PHYs», 2007.

15. Dart UWB Technology, https://www.zebra. $\mathrm{com} / \mathrm{us} / \mathrm{en} /$ products/location-solutions/dartuwb/dart-tag.html.

16. Trackit Systems, http://www.thetrackit.com/ RTLS.php.

17. Dw1000-product-brief.pdf, http://www. decawave.com/products/dwm1000-module.

18. Gubbi J, Buyya R, Marusic S, Palaniswami M. Internet of Things (Io'T): A vision, architectural elements, and future directions. Future Generation Computer Systems, 2013, 29:1645-1660.

19. Evans D. The Internet of Things. How the Next Evolution of the Internet Is Changing Everything. Cisco IBSG, White Paper, 2011:1-11. 